\title{
Child mortality inequalities across Rwanda districts: a geoadditive continuous-time survival analysis
}

\author{
François Niragire, ${ }^{1}$ Thomas N.O. Achia, ${ }^{2}$ Alexandre Lyambabaje, ${ }^{3,4}$ Joseph Ntaganira ${ }^{5}$ \\ ${ }^{1}$ Department of Applied Statistics, College of Business and Economics, University of Rwanda, Kigali, Rwanda; \\ ${ }^{2}$ School of Public Health, University of Witwatersrand, Johannesburg, South Africa; ${ }^{3}$ Department of \\ Human Nutrition and Dietetics, College of Medicine and Health Sciences, University of Rwanda, Kigali, \\ Rwanda; ${ }^{4}$ Inter-University Council for East Africa, Kampala, Uganda; ${ }^{5}$ Department of Epidemiology and \\ Biostatistics, College of Medicine and Health Sciences, University of Rwanda, Kigali, Rwanda
}

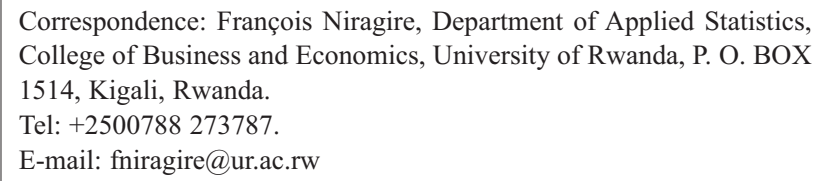

Key words: Spatial variation; Child mortality; Geoadditive model; Continuous-time hazard; Rwanda.

Acknowledgements: the authors are grateful to DHS Program for allowing them access to the 2010 RDHS data.

Contributions: FN, TNOA, AL, and JN contributed to the conception of the paper, writing and approval of the final version of the manuscript. FN obtained permission to use the 2010 RDHS data, conducted data analysis and wrote the first draft of the paper.

Conflict of interest: the authors declare no potential conflict of interest.

Ethical issues: the 2010 Rwanda Demographic and Health Survey protocol for data collection was reviewed and approved by the Rwanda National Ethics Committee, the Institutional Review Board of ICF International, and the Centres for Disease Control and Prevention (CDC) (NISR et al., 2011).

Funding: this research was supported by the Consortium for Advanced Research Training in Africa (CARTA). CARTA is jointly led by the African Population and Health Research Center and the University of the Witwatersrand and funded by the Wellcome Trust (UK) (Grant No: 087547/Z/08/Z), the Department for International Development (DfID) under the Development Partnerships in Higher Education (DelPHE), the Carnegie Corporation of New York (Grant No: B 8606), the Ford Foundation (Grant No: 1100-0399), Sida (Grant No: 54100029), and MacArthur Foundation Grant No: 10-95915-000-INP.

Received for publication: 11 January 2016.

Revision received: 21 October 2016.

Accepted for publication: 19 November 2016.

CCopyright F. Niragire et al., 2017

Licensee PAGEPress, Italy

Geospatial Health 2017; 12:450

doi:10.4081/gh.2017.450

This article is distributed under the terms of the Creative Commons Attribution Noncommercial License (CC BY-NC 4.0) which permits any noncommercial use, distribution, and reproduction in any medium, provided the original author(s) and source are credited.

\begin{abstract}
Child survival programmes are efficient when they target the most significant and area-specific factors. This study aimed to assess the key determinants and spatial variation of child mortality at the district level in Rwanda. Data from the 2010 Rwanda Demographic and Health Survey were analysed for 8817 live births that occurred during five years preceding the survey. Out of the children born, 433 had died before survey interviews were carried out. A full Bayesian geo-additive continuous-time hazard model enabled us to maximise data utilisation and hence improve the accuracy of our estimates. The results showed substantial district-level spatial variation in childhood mortality in Rwanda. District-specific spatial characteristics were particularly associated with higher death hazards in two districts: Musanze and Nyabihu. The model estimates showed that there were lower death rates among children from households of medium and high economic status compared to those from low-economic status households. Factors, such as four antenatal care visits, delivery at a health facility, prolonged breastfeeding and mothers younger than 31 years were associated with lower child death rates. Long preceding birth intervals were also associated with fewer hazards. For these reasons, programmes aimed at reducing child mortality gaps between districts in Rwanda should target maternal factors and take into consideration district-specific spatial characteristics. Further, child survival gains require strengthening or scaling-up of existing programmes pertaining to access to, and utilisation of maternal and child health care services as well as reduction of the household gap in the economic status.
\end{abstract}

\section{Introduction}

Global and regional disparities in child mortality have existed for many decades (UN, 2005, 2012, 2014). High child mortality rates have also remained a key indicator of low socioeconomic development or inadequate health care system (Reidpath and Allotey, 2003; Adebayo and Fahrmeir, 2005; Kwabena, 2011). Thus, levels of childhood mortality rates alone can indicate important differences in socioeconomic and health conditions between developed and developing countries. For example, the average under-five mortality for developed countries was 7 deaths per 1,000 live births in 2010 and 6 in 2012, whereas in developing countries it was about nine times higher: 63 in 2010 and 53 in 
2012, respectively (UN, 2012, 2014). Within a country, differences in childhood mortality are mostly attributed to unequal distribution, access to, or utilisation of, available resources that are critical to adequate child healthcare and nutrition (Adebayo and Fahrmeir, 2005; Ombok et al., 2010; Kwabena, 2011). The clustering of child deaths at the family or community levels has often been attributed to biological, environmental, demographic, socioeconomic, or health-related factors (Adebayo and Fahrmeir, 2005; Arulampalam and Bhalotra, 2006; Omariba et al., 2007; Mesike and Mojekwu, 2012; Niragire et al., 2011), as well as spatial characteristics of the geographic region in question (Adebayo and Fahrmeir, 2005; Kandala and Ghilagaber, 2006; Chin et al., 2011). In early 1980s, the analytical framework developed by Mosley and Chen (1984) provided more detailed explanation of the relationships among potential determinants of child mortality and the mechanisms by which they affect children's health. All potential factors that can affect a child's health status were classified as either socioeconomic (or distal) or proximate determinants (Mosley and Chen, 1984; Hill, 2003). The literature about childhood mortality and its determinants has grown rapidly after the declaration of the Millennium Development Goals (MDGs) in 2000 (UN, 2005). Research on child health outcome is also expected to multiply beyond the MDGs' period because of two main reasons. First, many countries have failed to achieve the MDG4 child survival target (UN, 2014). Second, the MDG achievements should be maintained or scaled-up in order to reach sustainable outcomes in the framework of the third goal (SDG 3) (UN, 2015).

There is an established consensus that improvement in child survival requires progress on multiple fronts beyond biomedical interventions (Mosley and Chen, 1984; Reidpath and Allotey, 2003; Adebayo and Fahrmeir, 2005; Kwabena, 2011). It is also recognised that child mortality determinants vary significantly across communities and countries, such that results for a country cannot be reliably generalised to another country (Black et al., 2003). Such determinants exist at the individual, household, and community levels (Mosley and Chen, 1984; Sastry, 1997; Arulampalam and Bhalotra, 2006; Niragire et al., 2011). In addition, child mortality determinants can be seen and followed very early, during pregnancy or at delivery (Ajaari et al., 2012; Singh et al., 2013), and their effects can carry on during advanced childhood ages.

The under-five mortality rate in Rwanda has remained around ten times higher than the average under-five mortality rates for developed countries (6 deaths per 1000 live births in 2012) (UN, 2005, 2012; NISR et al., 2011). In addition, the exceptionally rapid and sustained reduction in the child mortality rate over more than 10 years in Rwanda (152 deaths, 76 deaths and 50 deaths per 1,000 live births in 2005, 2010, and 2015 respectively) appears to mask important within-country variations (NISR et al., 2011; NISR, 2015). Previous studies of childhood mortality in Rwanda have mainly focused on socio-economic or proximate determinants (Niragire et al., 2011; Habimana-Kabano et al., 2015; Hong et al., 2009; Musafili et al., 2015). The only spatial analysis was conducted to compare child mortality risk factors between Rwanda and other two countries using merged census data (Kazembe et al., 2012). The results of the latter study are limited by the number of health and demographic variables readily available in utilised census data (Kazembe et al., 2012) as well as limitations associated with the measurement of child survival in months for a setting where neonatal mortality is high.

This study was initiated due to the need for more comprehen- sive and refined understanding of child mortality factors in Rwanda for prioritised, accelerated and efficient actions towards child survival. Such actions should target most influential or locally existing factors (Victora et al., 1997; Black et al., 2003; Ombok et al., 2010; Chin et al., 2011). The analysis presented here considers a larger number of potential factors of childhood mortality and uses an analytical model that includes neonatal mortality data.

\section{Materials and Methods}

\section{Study area}

Rwanda is a landlocked and mountainous country of 26,338 $\mathrm{km}^{2}$ situated between latitude $1^{\circ} 4$ ' and $2^{\circ} 51^{\prime} \mathrm{S}$ and longitude $28^{\circ} 63^{\prime}$ and $30^{\circ} 54^{\prime} \mathrm{E}$ (NISR et al., 2011). Rwanda shares borders with the Democratic Republic of Congo (DRC) to the west, Uganda to the north, Tanzania to the east and Burundi to the south (NISR et al., 2011). It is administratively divided into the City of Kigali and four provinces, which are in turn divided into 30 districts.

The population of Rwanda is predominantly rural, has an annual growth rate of $2.6 \%$ and is estimated to pass $12,000,000$ by the end of 2016. Rwanda has a high population density that increased from 321 to 416 persons $/ \mathrm{km}^{2}$ between 2002 and 2012 (NISR and MINECOFIN, 2014). In 2014, the proportion of the population below the national poverty line had dropped to 39.1 from 44.9\% in 2010 (NISR, 2015).

\section{Data}

The data used in this study are from the 2010 Rwanda Demographic and Health Survey (RDHS) that was implemented by the National Institute of Statistics of Rwanda (NISR) from September 2010 to March 2011 (NISR et al., 2011). Geographic data for the current administrative district boundaries of Rwanda were collected, processed and made available by the NISR in 2006 (NISR, 2006). The RDHS is conducted every five years and provides nationally representative estimates for various demographic, socio-economic, behavioural and health characteristics in Rwanda (NISR et al., 2011). During the 2010 RDHS, 13,671 women aged 15-49 (99.1\% response rate) and 6329 men aged $15-$ $59(98.7 \%$ response rate) were interviewed. In particular, data were gathered from the mothers for 9002 live births taking place within the last five years prior to the survey (NISR et al., 2011). Out of the children born, 518 had died by the interview dates. The database did not include 78 live births where the child died on day of birth, 47 live births of children born within the month of the survey and 60 children for whom there were missing data records on important covariates considered in this study. Thus, this study analysed data for 8,817 live births children with at least one-day survival and complete data records on considered variables, especially the survival time. Of these children, 433 had died before survey interviews were carried out.

\section{Analytic methods}

\section{Dependent variable}

This study adopted a Bayesian survival regression analysis (Hennerfeind et al., 2006) with the child survival time, measured as the number of survived days between child's birth and death or 
one month before the month of the survey, as the dependent variable. This variable was combined with a survival status indicator (Cox, 1972) taking on the value 0 if the survival time was censored and the value 1, if the child had died. For premature deaths, the survey recorded the age at death in days, months or years (NISR et al., 2011). The age of living children was collected in complete years only, and hence their survival time was calculated as a difference of dates of interview and child birth in century month codes (CMC) (Rutstein and Rojas, 2006).

In Rwanda, infant mortality constitutes an important component of the under-five mortality (NISR et al., 2011). Thus, unlike several previous studies (Adebayo and Fahrmeir, 2005; Kandala and Ghilagaber, 2006; Omariba et al., 2007) and following the recommendation of Adebayo and Fahrmeir (2005), the child survival time was measured in days by considering a standard 30days month in order to maximise the utilisation of the available mortality data.

\section{Covariates}

Mosley and Chen's (1984) analytical framework for the study of child mortality in developing countries and the existing literature (Adebayo and Fahrmeir, 2005; Kandala and Ghilagaber, 2006; Omariba et al., 2007; Chin et al., 2011) played a reference role in the selection of potential distal (demographic, socio-economic, cultural) and proximate factors of child mortality that were available in the 2010 RDHS data.

The selected set of eleven proximate determinants included four maternal factors namely: i) mother's age, ii) mother's fertility (number of living children born to this mother), iii) birth order, and iv) preceding birth interval; two health or illness control factors comprising (v) the number of antenatal care visits; and vi) place of delivery; four environmental factors comprising: vii) source of drinking water, viii) type of toilet facility used by household members, ix) type of fuel used for cooking or heating, and $\mathrm{x}$ ) whether the mother smoked tobacco. Factor (xi) duration of breastfeeding was the only one variable relevant to child nutrition.

The selected fifteen distal factors included the following six bio-demographic factors: i) child sex, ii) type of birth (multiplicity of birth), iii) household size, iv) sex of household head, v) age of household head, and vi) mother's marital status; one cultural factor: vii) mother's religion; and eight socioeconomic factors: viii) type of place of residence, ix) household's economic status, $\mathrm{x}$ ) mother's current employment status, xi) mother's occupation, and xii) partner's occupation, xiii) mother's educational level, xiv) partner's educational level, and xv) mother's exposure to mass media.

Most covariates were categorised with reference to the existing literature (Mosley and Chen, 1984; Adebayo and Fahrmeir, 2005; Omariba et al., 2007; JMP, 2008; Chin et al., 2011) in order to enable comparability across studies, whereas the classification of some other variables is self-explanatory.

Specifically, the exposure to mass media index was constructed according to the classification proposed by Fazle Rabbi (Rabbi, 2012). The household economic status variable was derived from the RDHS household wealth index (Rutstein and Johnson, 2004). The second and the third quintiles were grouped with the middle into a medium household economic status. All categorical covariates were dummy-coded and the first category was considered as the reference category to which other categories were compared.

\section{Statistical analysis}

The standard univariate Cox (1972) proportional hazards regression model was used in bivariate analyses to test for the association of each covariate with the child survival time at the $5 \%$ level of significance. Each dependent variable that showed a statistically significant relationship with the child survival time was considered for multivariable analysis based on a geoadditive continuous-time hazard regression model. Such survival models have been described in the literature (Hennerfeind et al., 2006; Kandala and Ghilagaber, 2006) and successfully applied in several studies (Adebayo and Fahrmeir, 2005; Kandala and Ghilagaber, 2006). For the particular application here, we considered a sample of $n$ live births and the set of obesrvations on these $i,\left(t_{i}, x_{i}, l_{i}\right.$, dis$\left.t_{i s}\right) ; s=1, \ldots, 30$; where $t_{i}=1, \ldots, 1799$ records the number of days the child $i$ survived (until death or end of the observation period); $x_{i}=\left(x_{i 1}, \ldots, x_{i p}\right)$ ' is a vector of observations on $p$ metrical covariates $X$, dist $t_{i s}$ is the administrative district $s$, where the child $i$ was living during the 2010 RDHS, and $l_{i}=\left(l_{i 1}, \ldots, l_{i q}\right)$ is a vector of $q$ observations on categorical covariates $L$. The geoadditive hazard regression model for the child $i$ residing in disttict $s$ is then defined by the equation (Eq. 1) according to Adebayo and Fahrmeir (2005) and Hennerfeind et al. (2006):

$h_{i}(t)=\exp \left[f_{0}(t)+f_{1}\left(x_{i 1}\right)+\ldots+f_{\mathrm{p}}\left(x_{i p}\right)+f_{\text {spat }}\left(\right.\right.$ dist $\left.\left._{i s}\right)+l_{i} \beta\right] \quad$ Eq. 1

Equation (2) indicates that (Eq. 1) can be written as $h_{i}(t)=\exp \left(\eta_{i}(t)\right)$ where $\eta_{i}(t)=\eta_{i}(t)$ is the geoadditive predictor:

$\eta_{i}(t)=f_{0}(t)+f_{1}\left(x_{i 1}\right)+\ldots+f_{\mathrm{p}}\left(x_{i p}\right)+f_{\text {spat }}\left(\right.$ dist $\left._{i s}\right)+l_{i} \beta$

Eq. 2

The baseline hazard rate $h_{0}(t)$ is reparameterised through $\exp \left(f_{0}(t)\right)$ where $f_{0}(t)=\log \left(h_{0}(t)\right)$ is the natural log-baseline effect. The smooth functions $f_{\mathrm{j}}($.$) represent nonlinear effects of metrical$ covariates $X$, which are modelled non-parametrically. The vector $\beta=\left(\beta_{0}, \beta_{1}, \ldots, \beta_{q}\right)$ is the vector of linear fixed-effect parameters for the categorical covariates, which were modelled in the usual parametric procedure (Hennerfeind et al., 2006). The effect of district of residence was modelled non-parametrically in order to adjust for small-area variation and neighbourhood property of spatial data interdependence (Hennerfeind et al., 2006; Flowerdew et al., 2008). The function $f_{\text {spat }}\left(\right.$ dist $\left._{s}\right)$ represents the spatial effect of district-specific (structured) and random (unstructured) characteristics and can be partitioned as using Equation 3 (Adebayo and Fahrmeir, 2005; Hennerfeind et al., 2006; Kandala and Ghilagaber, 2006):

$f_{\text {spat }}\left(\right.$ dist $\left._{s}\right)=f_{\text {str }}\left(\right.$ dist $\left._{s}\right)+f_{\text {unstr }}\left(\right.$ dist $\left._{s}\right)$

Eq. 3

For a full Bayesian statistical inference, prior distributions were specified for all model parameters and variances (Adebayo and Fahrmeir, 2005; Hennerfeind et al., 2006; Fahrmeir and Kneib, 2008). For fixed-effect parameter $\beta$ independent diffuse priors, $\pi(\beta) \propto_{c}$ ( $c$ is a constant) was assumed.

Nonparametric effects $f_{\mathrm{j}}(),. j=0.1, \ldots, p$ of the log-baseline hazard $f_{0}(t)=\log \left(h_{0}(t)\right)$, and metrical covariates $X$ were modelled through Bayesian penalised splines (P-splines) with partially improper random walk priors (Lang and Brezger, 2004; Hennerfeind et al., 2006; Fahrmeir and Kneib, 2008). Secondorder random walk Gaussian smoothness priors and cubic splines with twenty equidistant knots were considered in the present study (Hennerfeind et al., 2006; Fahrmeir and Kneib, 2008). 
Inverse-Gamma $I G\left(a_{j}, b_{j}\right)$ priors were assigned to all unknown variances $\tau^{2}{ }_{j}$ as specified in (Hennerfeind et al., 2006) where $a_{j}=b_{j}=0.001$ is a standard choice for a weakly informative prior.

The district-level structured spatial effect $f_{s t r}\left(\right.$ dist $\left._{s}\right)$ was modelled with Markov random field (MRF) prior because a spatial index of neighbouring districts was defined instead of exact spatial coordinates of the child's residence (Hennerfeind et al., 2006). The uncorrelated (random) district-level spatial effect $f_{\text {unst }}$ was assigned as independent and identically distributed Gaussian priors (Hennerfeind et al., 2006; Fahrmeir and Kneib, 2008). Districts were considered as neighbours if they share a boundary.

Among the candidate models, the model providing the best data fit was selected based on the deviance information criterion (DIC) statistic as described by Spiegelhalter et al. (2002) (where a smaller DIC statistic indicates better fit). Sensitivity analysis to the choice of prior parameters was carried out with different pairs of hyperparameter values $a$ and $b$ (Adebayo and Fahrmeir, 2005; Hennerfeind et al., 2006; Belitz et al., 2012). Fully Bayesian inference was carried out using Markov Chain Monte Carlo (MCMC) simulation (Brezger and Lang, 2006; Hennerfeind et al., 2006). Multivariate analyses and Bayesian inference and mapping were carried out using BayesX version 2.1 (Belitz et al., 2012). Inference was based on 1000 samples obtained through 5500 iterations with 500 burn-ins and 5 thinning.

\section{Results}

\section{Distribution of deaths among sampled live-birth chil- dren}

The results of this study are based on a sample of 8817 livebirth children, 433 of whom died during the five years preceding the survey. In total $358(82.7 \%)$ children died during their first year of life, including 191 (41.1\%) who died within their first month of life. Table 1 shows the district-level samples of live births and district-specific proportion of child deaths during the study period.

District-level samples of live births were based on the 2010 RDHS data and a code identifying each district was utilised to calculate the district-specific percent of child deaths. Musanze District showed the highest proportion (8.39\%), while Nyamasheke District registered the lowest (2.37\%). Figure 1

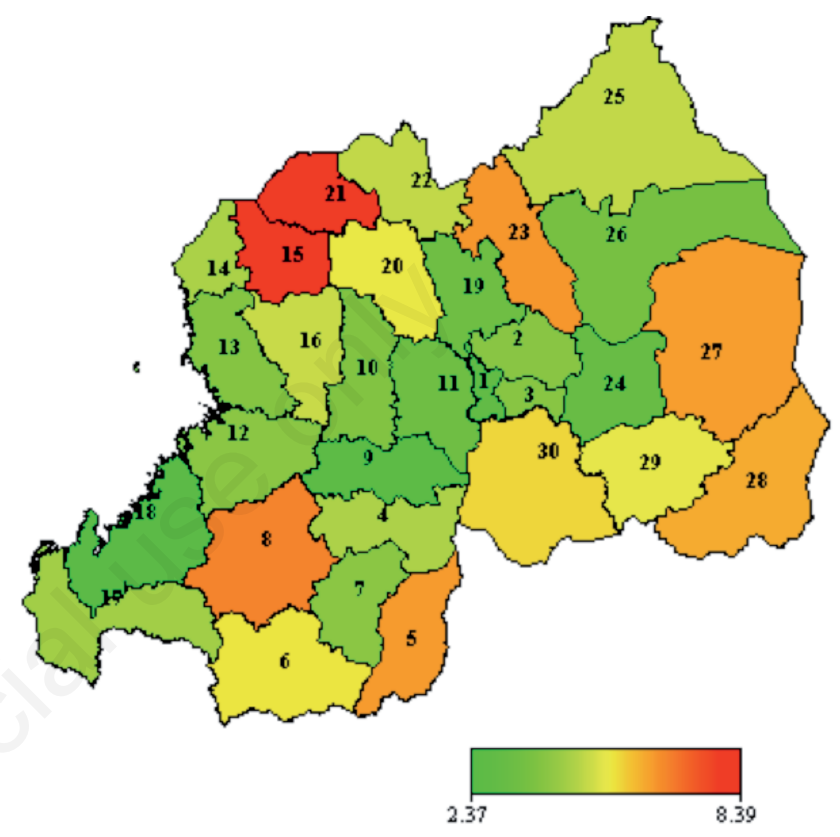

Figure 1. Variation of the district-level proportion of child deaths across Rwanda. The numeric values indicate the district codes (also referred to in Table 1).

Table 1. Distribution of live births and proportion of deaths among Rwandan districts.

\begin{tabular}{|c|c|c|c|c|c|c|c|c|c|}
\hline Code & District & $\mathrm{n}^{*}$ & Deaths & $\%$ & Code & District & $\mathrm{n}^{*}$ & Deaths & $\%$ \\
\hline 1 & Nyarugenge & 309 & 9 & 2.91 & 16 & Ngororero & 298 & 15 & 5.03 \\
\hline 2 & Gasabo & 344 & 14 & 4.07 & 17 & Rusizi & 310 & 14 & 4.52 \\
\hline 3 & Kicukiro & 320 & 13 & 4.06 & 18 & Nyamasheke & 295 & 7 & 2.37 \\
\hline 4 & Nyanza & 256 & 12 & 4.69 & 19 & Rulindo & 232 & 8 & 3.45 \\
\hline 5 & Gisagara & 324 & 21 & 6.48 & 20 & Gakenke & 290 & 16 & 5.52 \\
\hline 6 & Nyaruguru & 322 & 18 & 5.59 & 21 & Musanze & 286 & 24 & 8.39 \\
\hline 7 & Huye & 293 & 12 & 4.10 & 22 & Burera & 261 & 13 & 4.98 \\
\hline 8 & Nyamagabe & 279 & 19 & 6.81 & 23 & Gicumbi & 276 & 18 & 6.52 \\
\hline 9 & Ruhango & 234 & 6 & 2.56 & 24 & Rwamagana & 282 & 9 & 3.19 \\
\hline 10 & Muhanga & 231 & 9 & 3.90 & 25 & Nyagatare & 342 & 17 & 4.97 \\
\hline 11 & Kamonyi & 263 & 9 & 3.42 & 26 & Gatsibo & 327 & 12 & 3.67 \\
\hline 12 & Karongi & 268 & 11 & 4.10 & 27 & Kayonza & 280 & 18 & 6.43 \\
\hline 13 & Rutsiro & 326 & 13 & 3.99 & 28 & Kirehe & 303 & 19 & 6.27 \\
\hline 14 & Rubavu & 300 & 14 & 4.67 & 29 & Ngoma & 295 & 16 & 5.42 \\
\hline 15 & Nyabihu & 323 & 27 & 8.36 & 30 & Bugesera & 348 & 20 & 5.75 \\
\hline
\end{tabular}

${ }^{*}$ Children aged less than five years. The table is based on Rwanda Demographic and Health Survey (RDHS) data of 2010 ( $\mathrm{n}=8817$ live births). 
shows the distribution of the district-level percent of child deaths in map form. There is an oblique band of areas of low percent of child deaths between the districts of Nyamasheke and Gatsibo that stretches across the country from the West to the East. The southern region, which shares borders with Burundi and Tanzania, and the northern region, which shares border with Uganda, generally experienced higher proportions of district-level child deaths.

The frequency distributions of live births according to the survival status and the selected potential risk factors for child mortality were determined. For continuous covariates, the mean and the standard deviation (SD) are presented. In addition, a series of single-predictor Cox (1972) hazard regression model were fitted to data for each selected covariate in order to test its association with the child survival time at the $5 \%$ level of significance. Nonlinear relationships were also examined for metrical covariates (mother's age, duration of breastfeeding and age of household head) (Adebayo and Fahrmeir, 2005; Kandala and Ghilagaber, 2006). Through a single-covariate nonparametric Bayesian Cox hazard model, the child survival showed significant nonlinear relationship with the mother's age and duration of breastfeeding but not the age of household head.

Table 2 presents the frequency distributions of child deaths with relation to the factors that showed statistically significant associations with child survival. The data did not establish any sta- tistically significant linear relationship between child survival time and any of the following covariates (test $\mathrm{P}$ values within brackets): mother's age (0.257), child sex (0.372), sex of household head (0.453), age of household head (0.334), mother's marital status (0.350), mother's religion (0.327), place of residence $(0.645)$, mother's employment status (0.051), mother's occupation (0.268), partner's education (0.138), partner's occupation (0.141), mother's exposure to mass media (0.198), birth order (0.694), type of toilet facility (0.144), and type of cooking fuel used (0.521).

Statistically significant covariates were considered for multivariable analyses. Among the covariates that qualified for multivariable analyses, the highest percent missing data was calculated for preceding birth interval (0.35\%), a value considerably below $5 \%$. Thus, a complete-case multivariable data analysis was subsequently conducted (Schafer, 1999; Dong and Peng, 2013).

\section{Multivariable analyses}

\section{Selection of best fitting model}

Based on the data at hand, a set of nested geoadditive continuous-time hazard models were sequentially fitted to data. The fitted models were compared using DIC statistic (Spiegelhalter et al., 2002). Candidate models were labelled with the letters MD followed by a serial number in ascending order from one to five. The

Table 2. Distribution of child deaths in relation to the covariates that were significantly associated with child mortality.

\begin{tabular}{|c|c|c|c|c|}
\hline Factors & Factor levels & Alive & Dead & $\mathbf{P}$ \\
\hline Mother's age & Continuous & $30.63(6.59)$ & $31.26(7.14)$ & 0.257 \\
\hline Fertility & $\begin{array}{l}<3 \\
3-4 \\
\geq 5\end{array}$ & $\begin{array}{l}3536(40.10) \\
2767(31.38) \\
2081(23.60)\end{array}$ & $\begin{array}{l}241(2.73) \\
115(1.30) \\
77(0.87)\end{array}$ & $<0.001$ \\
\hline Preceding birth interval & $\begin{array}{l}\text { Short }(<24 \text { months }) \\
\text { Long }(\geq 24) \\
\text { First birth }\end{array}$ & $\begin{array}{l}1213(13.76) \\
5073(57.54) \\
2098(23.79)\end{array}$ & $\begin{array}{c}99(1.12) \\
223(2.53) \\
111(1.26)\end{array}$ & $<0.001$ \\
\hline Duration of breastfeeding & Continuous & $25.30(14.35)$ & $5.49(7.35)$ & $<0.001$ \\
\hline Antenatal visits & $\begin{array}{l}\text { No visit } \\
\quad<4 \\
\geq 4\end{array}$ & $\begin{array}{l}2432(27.58) \\
3787(42.95) \\
2165(24.55)\end{array}$ & $\begin{array}{l}277(3.14) \\
107(1.21) \\
49(0.56)\end{array}$ & $<0.001$ \\
\hline Place of delivery & $\begin{array}{c}\text { Home } \\
\text { Health facility }\end{array}$ & $\begin{array}{l}2572(29.17) \\
5812(65.92)\end{array}$ & $\begin{array}{l}175(1.98) \\
258(2.93)\end{array}$ & 0.001 \\
\hline Source of drinking water & $\begin{array}{l}\text { Improved } \\
\text { Other }\end{array}$ & $\begin{array}{l}5973(67.74) \\
2411(27.34)\end{array}$ & $\begin{array}{l}284(3.22) \\
149(1.69)\end{array}$ & 0.010 \\
\hline Mother smokes tobacco & $\begin{array}{l}\text { No } \\
\text { Yes }\end{array}$ & $\begin{array}{c}8026(91.03) \\
358(4.06)\end{array}$ & $\begin{array}{c}402(4.56) \\
31(0.35)\end{array}$ & 0.007 \\
\hline Type of birth & $\begin{array}{l}\text { Singleton } \\
\text { Multiple }\end{array}$ & $\begin{array}{c}8196(92.96) \\
188(2.13)\end{array}$ & $\begin{array}{c}392(4.45) \\
41(0.47)\end{array}$ & $<0.001$ \\
\hline Household size & $\begin{array}{l}\leq 5 \\
>5\end{array}$ & $\begin{array}{l}4653(52.77) \\
3731(42.32)\end{array}$ & $\begin{array}{l}284(3.21) \\
149(1.69)\end{array}$ & $<0.001$ \\
\hline Economic status & $\begin{array}{c}\text { Low } \\
\text { Medium } \\
\text { High }\end{array}$ & $\begin{array}{l}1925(21.83) \\
4954(56.19) \\
1505(17.07)\end{array}$ & $\begin{array}{l}122(1.38) \\
250(2.84) \\
61(0.69)\end{array}$ & 0.013 \\
\hline Mother's education & $\begin{array}{l}\text { No school education } \\
\text { Primary } \\
\text { Secondary or higher }\end{array}$ & $\begin{array}{c}1559(17.68) \\
6029(68.38) \\
796(9.03)\end{array}$ & $\begin{array}{c}109(1.24) \\
287(3.26) \\
37(0.42)\end{array}$ & 0.004 \\
\hline
\end{tabular}

Values are expressed as mean (standard deviation), presented for metrical covariates. 
variable dist stands for district, and the terms proximate and background hold places for all model terms involving proximate and background factors respectively, i.e.:

proximate $=$ mother's age + breastfeeding duration + fertility + preceding birth interval + antenatal visit + place of delivery + source of drinking water + tobacco smoking status

background $=$ type of birth + household size + economic status + mother's education

The geoadditive predictors $\eta_{i}$ of the four candidate models for an individual child $i, i=1, \ldots, 8817$; residing in district $s, s=1, \ldots, 30$ were specified as follows:

MD1: $\eta_{\text {is }}\left(t_{i}\right)=f_{0}\left(t_{i}\right)+f_{\text {unstr }}\left(\right.$ dist $\left._{s}\right)$

MD2: $\eta_{\text {is }}\left(t_{i}\right)=f_{0}\left(t_{i}\right)+f_{\text {str }}\left(\right.$ dist $\left._{s}\right)+f_{\text {unstr }}\left(\right.$ dist $\left._{s}\right)$

MD3: $\eta_{\text {is }}\left(t_{i}\right)=f_{0}\left(t_{i}\right)+f_{\text {str }}\left(\right.$ dist $\left._{s}\right)+f_{\text {unstr }}\left(\right.$ dist $\left._{s}\right)+$ background $_{i}+$ proximate $_{i}$

MD4: $\eta_{i s}\left(t_{i}\right)=f_{0}\left(t_{i}\right)+$ background $_{i}+$ proximate $_{i}$ (without spatial effect terms)

Model MD1 was fitted under the assumption that an eventual clustering of district-level child deaths was only due to spatial random effects. MD3 adjusted the spatial effects by the effect of distal and proximate factors and vice-versa. Finally, MD4 assessed the added advantage of adopting a spatial modelling methodology.

The results showed that model MD3 (DIC=6152.347) provided the best data fit. The DIC statistics were 8456.847, 8441.346 and 6216.702 for MD1, MD2 and MD4, respectively. Therefore, this study results were based on the geoadditive model MD3, which comprised relevant distal and proximate factors.

\section{Outcome of the geoadditive continuous-time hazard model}

\section{Linear fixed effects}

Based on the posterior mean estimates of fixed-effects parameters for categorical covariates with corresponding standard error, 95 and $80 \%$ point-wise credible intervals (CIs), the posterior mean hazards ratios (PHR) were derived along with their respective $95 \%$ CIs (Table 3). The results show that the following five fixed-effect categorical covariates were statistically significant determinants of child mortality in Rwanda (95\% CI does not include unity): i) economic status; ii) fertility; iii) preceding birth interval, iv) antenatal visits, and v) place of delivery. The household's economic status was the only significant distal determinant of the child mortality. The data indicated that, compared to children living in low-economic status households, the PHR was $41 \%$ and $38 \%$ lower among children living in households with medium and high economic status, respectively. The PHR was $88 \%$ lower among children born to mothers who had more than 4 children and 73\% lower among children born to mothers who had between three and four children.

The other three significant fixed-effect determinants can be classified as heath factors. The hazard rate was 30\% lower among children delivered at a health facility compared to those delivered elsewhere. The death rate was 79 and $83 \%$ lower among children whose mothers achieved less than four antenatal visits and four antenatal visits or more, respectively, compared to children whose mothers did not attend any antenatal care. Compared to live births with the preceding birth interval less than 24 months, the PHRs were 27 and $54 \%$ lower among live births with a preceding birth interval of 24 months or above and the child being the first born, respectively.
Among the covariates that were not statistically significant determinants of child mortality, the mother's education showed the largest differences in posterior hazard ratios, i.e. $12 \%$ lower among those born to a mother who had attained secondary or higher education, and 9\% lower among those born to mothers who had only attained primary education compared with children born to mothers who did not complete any formal education at all.

\section{Non-linear effects}

These effects were significant for mother's age and duration of breastfeeding. The posterior mean estimates of the effect of the mother's age and corresponding CIs (Figure 2A) showed that mothers younger than 32 years were significantly associated with lower rates of child death (strictly negative CIs). Mothers older than 40 years significantly contributed to an increase of child death rate (strictly positive $\mathrm{CIs}$ ). There were also wide, strictly negative CIs of an increasing effect of mothers aged less than 21 years. From 40 years, the effect of the mother's age became positive, statistically significant and increased sharply until the age of 49 years.

Expectedly, children who were breastfed for less than two years (24 months) were significantly associated with higher death rates as shown in Figure 2B. The posterior mean effects were positive with strictly positive $95 \%$ CIs. Breastfeeding for more than 27 months was associated with lower hazard rates which remained

Table 3. Posterior hazard ratios with $95 \%$ credible intervals for the linear effects.

\begin{tabular}{|c|c|c|c|c|}
\hline Covariate & $\begin{array}{l}\text { Reference } \\
\text { category }\end{array}$ & PHR & $2.5 \%$ & $97.5 \%$ \\
\hline Household size & $\begin{array}{l}\leq 5 \\
>5\end{array}$ & $\begin{array}{l}1.00 \\
1.05\end{array}$ & $\begin{array}{c}- \\
0.82\end{array}$ & $\begin{array}{c}- \\
1.34\end{array}$ \\
\hline Birth type & $\begin{array}{c}\text { Single } \\
\text { Multiple }\end{array}$ & $\begin{array}{l}1.00 \\
1.04\end{array}$ & 0. & $\begin{array}{c}- \\
1.55\end{array}$ \\
\hline Economic status & $\begin{array}{l}\text { Low } \\
\text { Medium } \\
\text { High }\end{array}$ & $\begin{array}{l}1.00 \\
0.59 \\
0.62\end{array}$ & $\begin{array}{c}- \\
0.46 \\
0.41\end{array}$ & $\begin{array}{l}- \\
0.74 * \\
0.92 *\end{array}$ \\
\hline Mother's education & $\begin{array}{c}\text { No education } \\
\text { Primary } \\
\text { Secondary and higher }\end{array}$ & $\begin{array}{l}1.00 \\
0.91 \\
0.88\end{array}$ & $\begin{array}{c}- \\
0.71 \\
0.55\end{array}$ & $\begin{array}{c}- \\
1.19 \\
1.41 \\
\end{array}$ \\
\hline Source of drinking water & $\begin{array}{c}\text { Improved } \\
\text { Other sources }\end{array}$ & $\begin{array}{l}1.00 \\
1.00\end{array}$ & 0.80 & $\begin{array}{c}- \\
1.28\end{array}$ \\
\hline Mother smokes tobacco & $\begin{array}{l}\text { No } \\
\text { Yes }\end{array}$ & $\begin{array}{l}1.00 \\
0.95\end{array}$ & $\overline{-}$ & $\begin{array}{c}- \\
1.44\end{array}$ \\
\hline Fertility & $\begin{array}{l}<3 \text { children } \\
3-4 \text { children } \\
>4 \text { children }\end{array}$ & $\begin{array}{l}1.00 \\
0.27 \\
0.12\end{array}$ & $\begin{array}{c}- \\
0.21 \\
0.08\end{array}$ & $\begin{array}{c}- \\
0.37^{*} \\
0.18^{*}\end{array}$ \\
\hline Preceding birth interval & $\begin{array}{l}<24 \text { months } \\
\geq 24 \text { months } \\
\text { First birth }\end{array}$ & $\begin{array}{l}1.00 \\
0.73 \\
0.46\end{array}$ & $\begin{array}{c}- \\
0.56 \\
0.33\end{array}$ & $\begin{array}{c}- \\
0.95^{*} \\
0.65^{*}\end{array}$ \\
\hline Antenatal visits & $\begin{array}{l}\text { No visit } \\
<4 \text { visits } \\
\geq 4 \text { visits }\end{array}$ & $\begin{array}{l}1.00 \\
0.21 \\
0.17\end{array}$ & $\begin{array}{c}- \\
0.16 \\
0.12\end{array}$ & $\begin{array}{c}- \\
0.28^{*} \\
0.24 *\end{array}$ \\
\hline Place of delivery & $\begin{array}{c}\text { Home } \\
\text { Health facility }\end{array}$ & $\begin{array}{l}1.00 \\
0.70\end{array}$ & $\overline{-}-$ & $\begin{array}{c}- \\
0.89 *\end{array}$ \\
\hline
\end{tabular}

PHR, posterior hazard ratio. *Statistically significant. PHRs and their corresponding confidence intervals provide a measure of magnitude of each covariate's effect in addition to its significance and direction. 
statistically not significant until the $49^{\text {th }}$ month. Those children who were breastfed for more than 49 months were associated with statistically significant lower death rates. The CIs became wider beyond the $58^{\text {th }}$ month when the effects ceased to be statistically significant. The baseline effect, presented on Figure $2 \mathrm{C}$, declined slowly and unsteadily until 1790 days (59 months). Thus, these children died faster during infancy than in childhood.

\section{Unadjusted spatial effects}

These effects are shown in Figure 3. The unadjusted unstructured spatial effect had weak influence on total spatial effect (Figure 3A). The patterns of the unadjusted total spatial effects and structured spatial effect were similar (Figure 3B), but these maps were slightly different from the one depicting percentages of child deaths in Figure 1. The pattern of the total spatial effect was dominated by the structured spatial effect. However, none of the district-level unadjusted spatial effects were statistically significant, not even with $80 \%$ CIs.

\section{Adjusted spatial effects}

The posterior mean estimates of the district-level structured spatial effects are presented in the map in Figure 4A, which illustrates the pattern and difference in magnitude of the effects. Data showed that structured effects are higher in the north, and easternsouth of Rwanda. The results showed no statistically significant difference in structured spatial effects on child mortality rates across the Rwanda districts with 95\% CIs. Figure 4B, however, indicates that the district level structured spatial effect was associated with higher child mortality rate in districts of Musanze and Nyabihu (black) when the CIs were narrowed down to $80 \%$ probability, whereas they were associated with lower child mortality rate in the districts of Nyamasheke, Muhanga, and Gasabo (white). The adjusted unstructured spatial effect showed distinctive spatial patterns, but was not even statistically significant with $80 \%$ CIs.

The posterior mean estimates of the total spatial effect for each district are visualised on the map on Figure 5A. The 95\% map of posterior probability for total spatial effect is presented on Figure 5B. Data revealed that higher child death rate was associated with living in the districts of Gisagara, Musanze, Nyabihu, Gicumbi, and Nyagatare. Lower child mortality rate was associated with the districts of Nyamasheke, Muhanga, Gasabo, Rwamagana, and Gatsibo. The significance of the total spatial effects expands to more districts when the $80 \%$ CIs are considered. In sum, the results indicated that failure to take into consideration the spatial location would negatively affect the childhood mortality risk estimates for Rwanda.

\section{Sensitivity analysis}

Based on model MD3, we operated changes in the values of hyperparameter $a$ and $b$, and examined corresponding changes in the variances of the estimated non-linear effects, as shown in Table 4 . In addition to the default couple ( $a=0.001, b=0.001$ ), the following couples of values for $a$ and $b$ were considered: ( $\mathrm{a}=0.0001$, $\mathrm{b}=0.0001) ;(\mathrm{a}=0.01, \mathrm{~b}=0.01)$; and $(\mathrm{a}=0.001, \mathrm{~b}=0.0005)$ (Adebayo and Fahrmeir, 2005; Hennerfeind et al., 2006; Belitz et al., 2012). The results in Table 4 showed that the variances of the estimated splines for continuous covariates and spatial components remained practically stable with respect to important changes in the parameters.

\section{Discussion}

The district level structured and total effects of spatial characteristics were significant determinants of child mortality. Nonspatial determinants were dominated by proximate determinants where economic status of the household was the only significant distal determinant. In general, the mortality rates were higher in the North and in Southeastern Rwanda where the districts are predominantly rural.

A

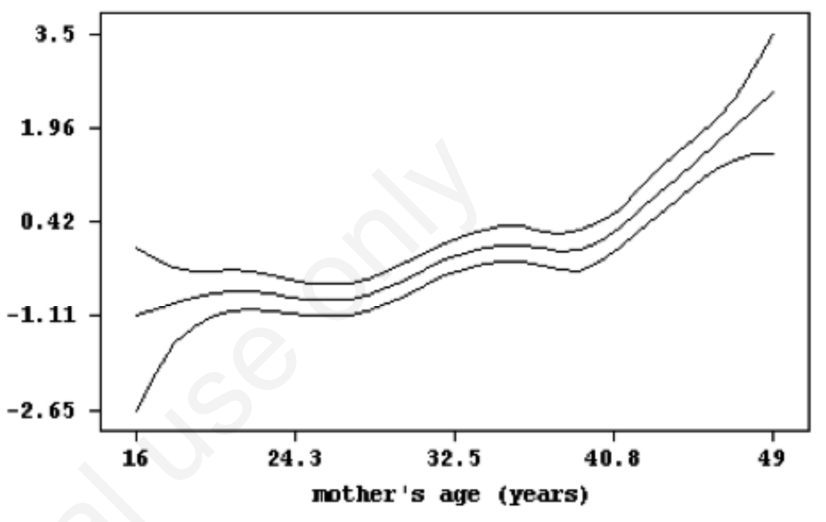

B

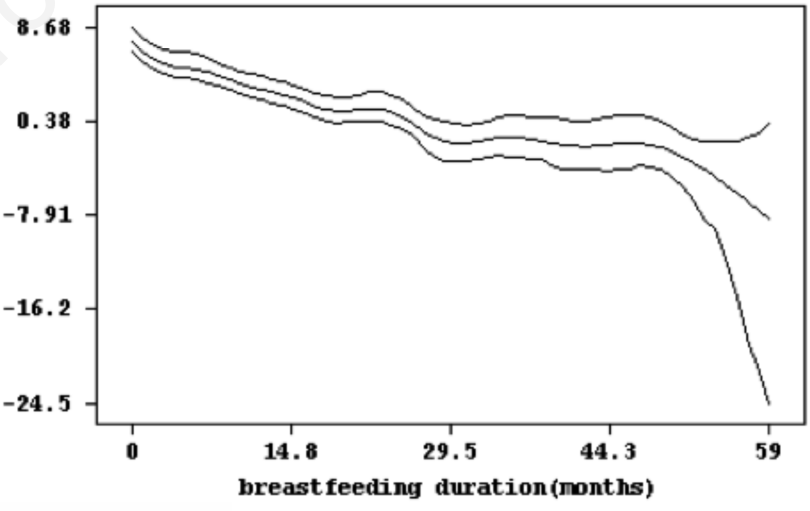

C

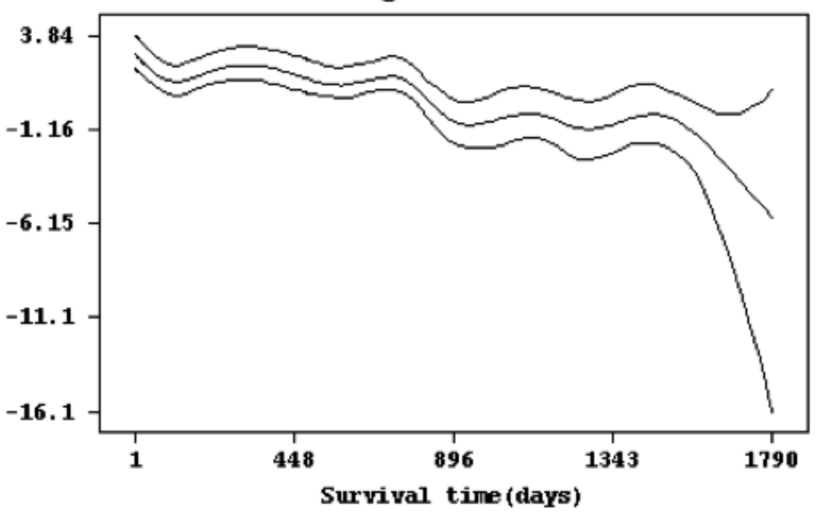

Figure 2. Non-linear effects of mother's age (A), duration of breastfeeding (B) and survival time (C) with $95 \%$ credible intervals. 
Small-area variations in childhood mortality have also been found in several other countries (Adebayo and Fahrmeir, 2005; Kandala and Ghilagaber, 2006; Chin et al., 2011). The important spatial characteristics that are critical to child mortality reflect the status of the household environment as determined by source of water, toilet facility, air pollution, hygienic breastfeeding, etc. (Macassa et al., 2004; Adebayo and Fahrmeir, 2005). They also include community and individual factors that influence access to health care services, availability and regularity of a nutritionally adequate child's diet, which are more predominant in certain areas than in the others (Kandala and Ghilagaber, 2006; Ombok et al., 2010; Schoeps et al., 2011).

The rural household environment is more likely to be less favourable to a child's life compared to the urban environment (Mesike and Mojekwu, 2012). In particular, the altitudinal heights that reach more than $2000 \mathrm{~m}$ (NISR et al., 2011) in the North limit access to safe water sources and health care institutions in some areas and can hence affect the quality of child health care (Schoeps et al., 2011).

Expectedly, maternal factors (age, fertility, birth interval) as well as disease prevention and treatment factors (place of delivery, antenatal care visits) dominated the set of proximate determinants of child mortality in Rwanda. Proximate determinants generally exert their greatest influence during infancy although their effects can carry on beyond infancy (Omariba et al., 2007). Several studies have found similar results elsewhere (Ajaari et al., 2012; Singh et al., 2013). The results for the baseline effect reaffirmed that the majority of child deaths in Rwanda occurred during the first year of life (NISR et al., 2011).

In Rwanda, the effect of the improvement in household economic status from low to high level was associated with a $33 \%$ decrease in posterior hazards ratio. The latter decreased by $42 \%$ when economic status of the household improved from low to medium levels. Several studies reported similar findings (Kandala

Table 4. Sensitivity analysis based on the best fitting model.

\begin{tabular}{|c|c|c|c|c|}
\hline Effects & $a=0.0001, b=0.0001$ & $a=0.001, b=0.001$ & $a=0.01, b=0.01$ & $a=0.001, b=0.0005$ \\
\hline $\mathbf{T}^{2} \log ($ baseline $)$ & $10.307(6.517)$ & $3.525(2.070)$ & $10.42(9.904)$ & $11.032(9.550)$ \\
\hline $2.5-97.5 \%$ & $1.146,26.921$ & $0.385,8.854$ & $0.438,34.682$ & $1.044,35.214$ \\
\hline $\begin{array}{l}\mathrm{T}^{2} \text { mother's age } \\
2.5-97.5 \%\end{array}$ & $\begin{array}{c}0.096(0.141) \\
0.006,0.433\end{array}$ & $\begin{array}{c}0.108(0.129) \\
0.006,0.455\end{array}$ & $\begin{array}{c}0.132(0.148) \\
0.012,0.547\end{array}$ & $\begin{array}{c}0.102(0.148) \\
0.006,0.460\end{array}$ \\
\hline $\begin{array}{l}\mathrm{T}_{\text {duration_of_breastfeeding }} \\
2.5-97.5 \%\end{array}$ & $\begin{array}{c}4.588(4.539) \\
0.477,16.501\end{array}$ & $\begin{array}{c}6.304(6.012) \\
0.485,22.245\end{array}$ & $\begin{array}{l}6.095(7.799) \\
0.131,24.691\end{array}$ & $\begin{array}{c}7.126(6.666) \\
0.555,25.556\end{array}$ \\
\hline $\begin{array}{l}\mathrm{T}^{2} \text { str } \\
2.5-97.5 \%\end{array}$ & $\begin{array}{c}0.736(0.646) \\
(0.018,2.367\end{array}$ & $\begin{array}{c}0.751(0.550) \\
0.007,1.954\end{array}$ & $\begin{array}{c}0.387(0.471) \\
0.006,1.592\end{array}$ & $\begin{array}{c}0.918(0.633) \\
0.012,2.308\end{array}$ \\
\hline $\begin{array}{l}\mathrm{T}^{2}{ }_{\text {unstr }} \\
2.5-97.5 \%\end{array}$ & $\begin{array}{c}0.171(0.168) \\
0.000,0.554\end{array}$ & $\begin{array}{c}0.138(0.153) \\
0.001,0.552\end{array}$ & $\begin{array}{c}0.317(0.175) \\
0.036,0.734\end{array}$ & $\begin{array}{c}0.128(0.148) \\
0.0004,0.495\end{array}$ \\
\hline
\end{tabular}
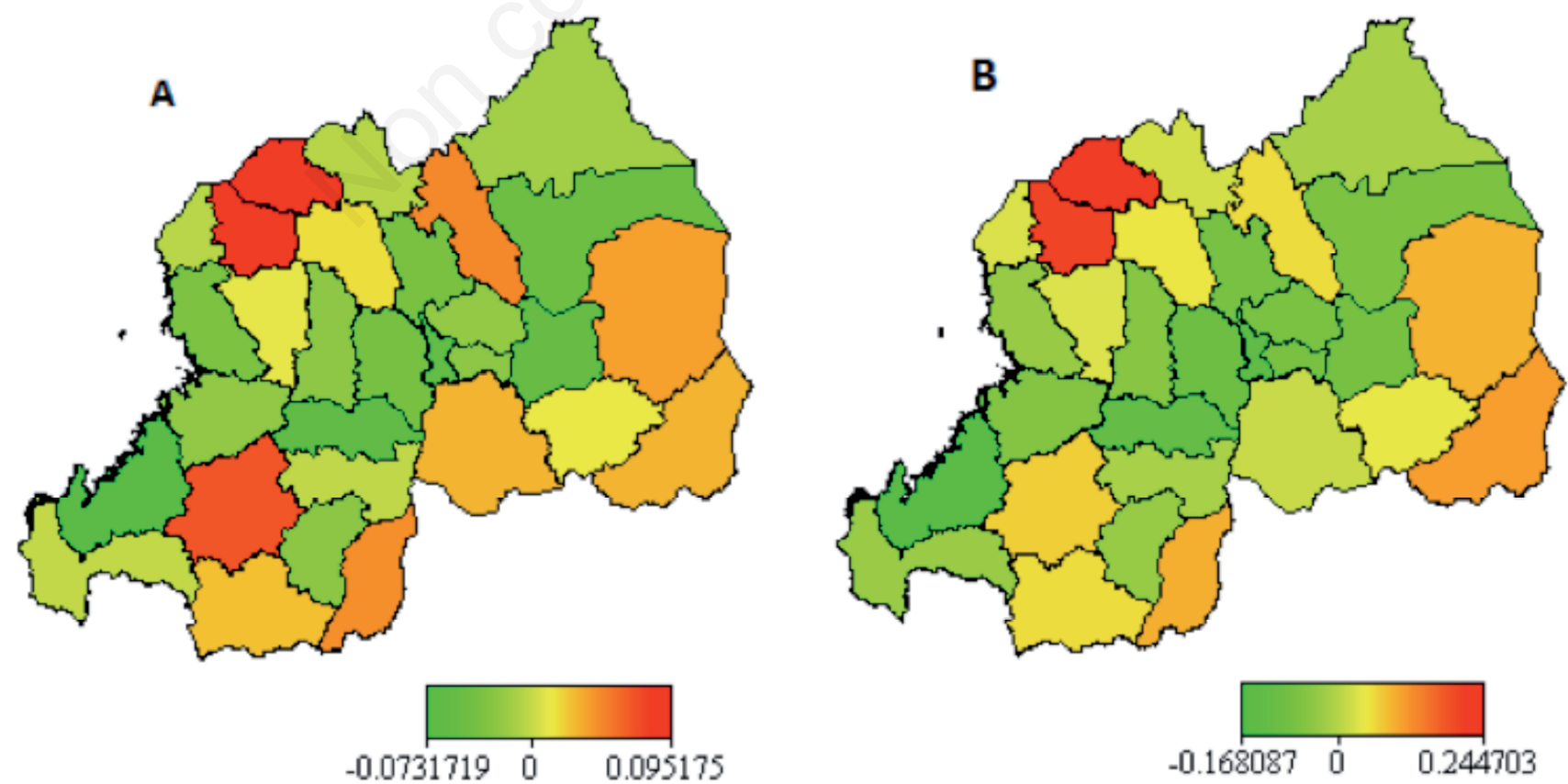

Figure 3. Posterior mean of the unstructured (A) and total (B) unadjusted district level spatial effects. 
and Ghilagaber, 2006; Hong et al., 2007; Kwabena, 2011). Importantly, children in medium or high economic status households can have easier access to a nutritionally adequate food, timely and improved health or medical care as well as a safe and stimulating household environment (Macassa et al., 2004; Adebayo and Fahrmeir, 2005). In the particular context of Rwanda, the persistent effect of the household's economic status on the under-five's mortality was mainly exercised by depriving the poor of adequate nutrition rather than access to health care services because of two important facts. First, the majority of households were beneficiaries of the Community Based Health Insurances (CBHIs) scheme. Combined with a stimulated participation of the Community Health Workers (CHWs), CBHIs have increased access to and utilisation of child and maternal health
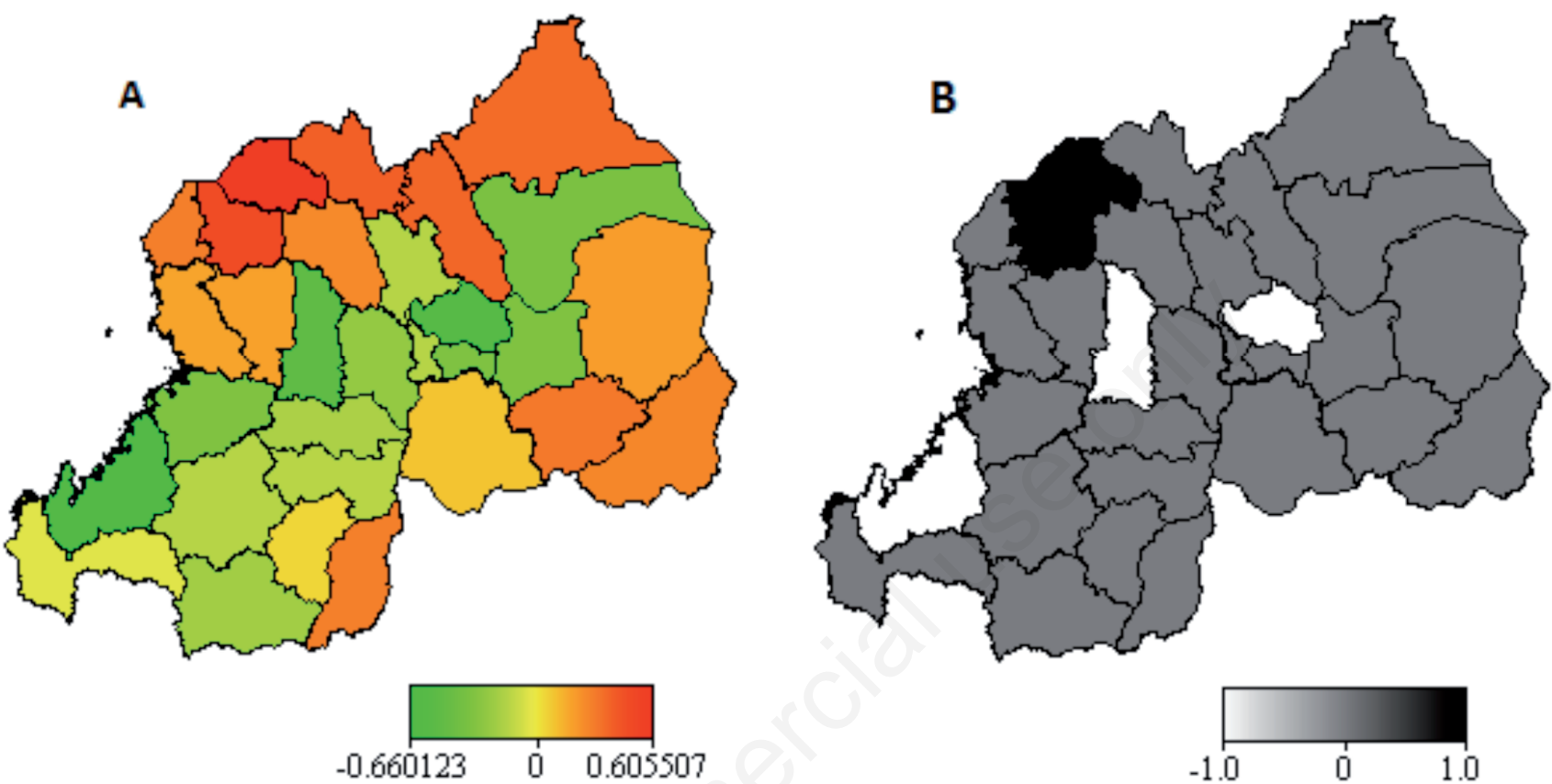

Figure 4. Posterior mean of the adjusted structured spatial effect (A) and corresponding posterior $80 \%$ probability map (B).
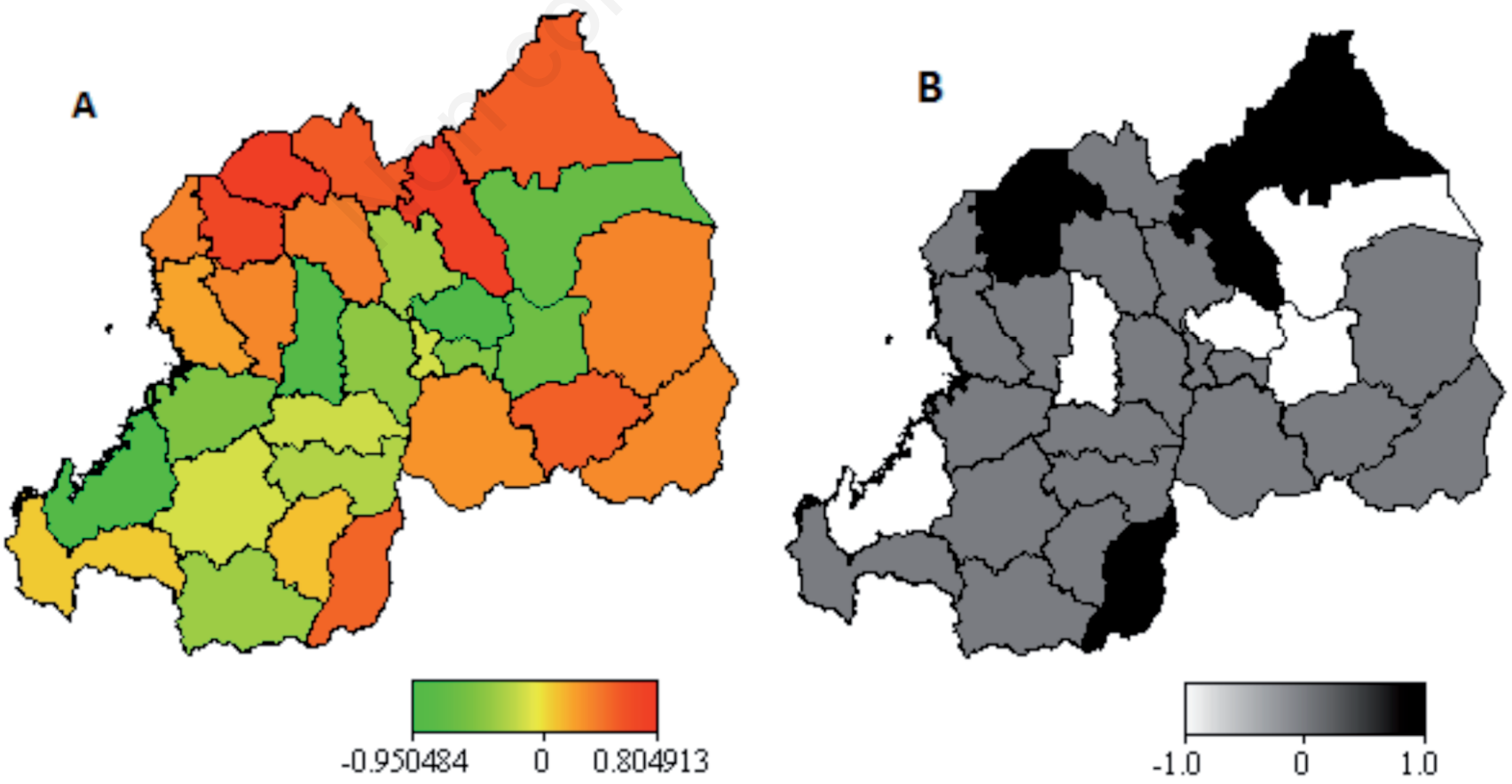

Figure 5. Posterior mean of the adjusted total spatial effect (A) and corresponding posterior $95 \%$ probability map (B). 
care services among all economic classes in Rwanda (Saksena et al., 2011; Sekabaraga et al., 2011; Lu et al., 2012). Second, 44\% of the under-five children were found stunted and $11 \%$ underweight in Rwanda in 2010 (NISR et al., 2011).

Birth spacing was found to be a significant determinant of child mortality in Rwanda. The negative effect of short birth intervals on maternal and child mortality and morbidity has been documented (Orji et al., 2004; Norton, 2005). Competition for nutrition and care among siblings of comparable ages also contribute to excess child deaths due to short preceding birth (Hong et al., 2009). Women who had fewer children were associated with increased risk of losing a child compared to other women. The results do not suggest that high fertility increased child survival in Rwanda, but only that higher child death rates occurred among women who reported small numbers of living children. The results support previous findings of clustered child mortality at mother level (Arulampalam and Bhalotra, 2006; Niragire et al., 2011).

This study showed that attending antenatal care consultations, or delivery at a health facility, were significantly associated with lower rates of child death. Several studies arrived at the same conclusion (Adebayo and Fahrmeir, 2005; Ajaari et al., 2012). The effects of the two proximate determinants can carry on during the whole childhood period (Omariba et al., 2007; Ajaari et al., 2012).

Mother's education was not a significant determinant of child mortality. However, there were relatively large differences in hazard ratios between the levels of the mother's education. In the context of Rwanda, the usual effect of the mother's education might have been significantly attenuated by a combination of improved access to medical health care services through CBHIs (Lu et al., 2012; Nyandekwe et al., 2014) and improved utilisation of child and maternal health care services thanks to stimulated efforts of CHWs (Sekabaraga et al., 2011).

The effect of the mother's age contributed to slowly increasing hazard rates. Mother's age have been associated with biological factors that determine the pregnancy or delivery complications or outcome for women below twenty (teenage pregnancy) or beyond thirty-five (advanced maternal age) (Luke and Brown, 2007; Selemani et al., 2014). There is also a significant decline in child health care due to reduction in mother's physical energy during advanced age (Sutcliffe et al., 2012). Further, a teen mother may lack sufficient experience in child's health care and adequate feeding (Selemani et al., 2014).

Expectedly, breastfeeding for less than 24 months was associated with a significantly higher rate of child death. Several previous studies have also found similar results (Adebayo and Fahrmeir, 2005; Mané et al., 2006; Girma and Berhane, 2011; Kwabena, 2011). In spite of the high risk of HIV transmission during breastfeeding (Miotti et al., 1999; Nduati et al., 2000), exclusive breastfeeding for first 6 months of child life dominated various recommendations (WHO, 2013).

Despite the strengths of this study, which mainly lie in its methodology, there is a need to highlight limitations that emanate from the analysed data. Cross-sectional survey data cannot establish the cause or effect between variables. Retrospective survey data also suffer from recall bias that affects the reported dates of births or deaths of children (Omariba et al., 2007; NISR et al., 2011). Children's data from DHS are collected from their respective living mothers (NISR et al., 2011). Hence, there was a selection bias following the omission from birth histories of those children, whose mothers were no longer alive. Nonetheless, the anal- ysed data remain nationally representative and the results are valid and generalisable to the whole country (NISR et al., 2011). Most of factors not captured in the analysis such as nutrition, and immunization status are not generally collected for children who died prior to data collection. These factors can largely be linked to spatial characteristics assessed in this study.

\section{Conclusions}

A structured geoadditive continuous-time hazard model permitted improved utilisation of child mortality data in Rwanda where most of child deaths occurred during the first year of life. There is evidence of significant differences in under-five children's mortality between districts in Rwanda that can be attributed to the effect of spatial characteristics. There is also a sign of clustering of child mortality related to attributes associated with the mothers. The results suggest that, despite exceptional reduction in national child mortality rate in Rwanda, the district-level disparities remain an important problem of health equity. Thus, child survival programmes tailored to local risk factors connected with child mortality would be more efficient in Rwanda. The persistent effect of the household economic status indicates that reduction of the gaps between economic classes in Rwanda is crucial for a significant reduction in child mortality inequality.

\section{References}

Adebayo SB, Fahrmeir L, 2005. Analysing child mortality in Nigeria with geoadditive discrete-time survival models. Stat Med 24:709-28.

Ajaari J, Masanja H, Weiner R, Abokyi SA, Owusu-Agyei S, 2012. Impact of place of delivery on neonatal mortality in rural Tanzania. Int J MCH AIDS 1:49-59.

Arulampalam W, Bhalotra S, 2006. Sibling death clustering in India: state dependence versus unobserved heterogeneity. J Roy Stat Soc A 169:829-48.

Belitz C, Brezger A, Kneib T, Lang S, 2012. BayesX: software for Bayesian inference in structured additive regression models. Version 2.0.1. Methodology manual. University of Munich, Munich, Germany.

Black R, Morris S, Bryce J, 2003. Where and why are 10 million children dying every year? Lancet 361:2226-34.

Brezger A, Lang S, 2006. Generalized structured additive regression based on Bayesian P-splines. Comput Stat Data Anal 50:967-91

Chin B, Montana L, Basagana X, 2011. Spatial modeling of geographic inequalities in infant and child mortality across Nepal. Health Place 17:929-36.

Cox DR, 1972. Regression models and life-tables. J Roy Stat Soc B 34:187-220.

Dong Y, Peng C-YJ, 2013. Principled missing data methods for researchers. Springerplus 2:222.

Fahrmeir L, Kneib T, 2008. Propriety of posteriors in structured additive regression models: theory and empirical evidence. J Stat Plan Inference 139:843-59.

Flowerdew R, Manley D, Sabel C, 2008. Neighbourhood effects on health: does it matter where you draw the boundaries? Soc Sci Med 66:1241-55.

Girma B, Berhane Y, 2011. Children who were vaccinated, breast 
fed and from low parity mothers live longer: a community based case-control study in Jimma, Ethiopia. BMC Public Health 11:197.

Habimana-Kabano I, Broekhuis A, Hooimeijer P, 2015. The effect of pregnancy spacing on fetal survival and neonatal mortality in Rwanda: a Heckman selection analysis. J Biosoc Sci 48:358-73.

Hennerfeind A, Brezger A, Fahrmeir L, 2006. Geoadditive survival models. J Am Stat Assoc 101:1065-75.

Hill K, 2003. Frameworks for studying the determinants of child survival. B World Health Organ 81:138-9.

Hong R, Ayad M, Rutstein S, Ren R, 2009. Childhood mortality in Rwanda: levels, trends, and differentials; further analysis of the Rwanda demographic and health surveys 1992-2007/08. DHS Further Analysis Reports No. 66. ICF Macro, Calverton, MD, USA.

Hong R, Mishra V, and Michael J, 2007. Economic disparity and child survival in Cambodia. Asia Pac J Public Health 19:37-44.

JMP, 2008. Progress on drinking water and sanitation: special focus on sanitation. WHO Library Cataloguing-in-Publication Data, UNICEF and WHO, Geneva and New York, Switzerland and USA.

Kandala N-B, Ghilagaber G, 2006. A geo-additive Bayesian discrete-time survival model and its application to spatial analysis of childhood mortality in Malawi. Quality Quantity 40:935-57.

Kazembe L, Clarke A, Kandala N-B, 2012. Childhood mortality in sub-Saharan Africa: cross-sectional insight into small-scale geographical inequalities from Census data. BMJ Open 2:e001421.

Kwabena AK, 2011. Socio-economic factors affecting under five mortality in South Africa: an investigative study. JETEMS 2:104-10.

Lang S, Brezger A, 2004. Bayesian P-Splines. J Comput Graph Stat 13:183-212.

Lu C, Chin B, Lewandowski JL, Basinga P, Hirschhorn LR, Hill K, Murray M, Binagwaho A, 2012. Towards universal health coverage: an evaluation of Rwanda mutuelles in its first eight years. PLoS One 7:e39282.

Luke B, Brown MB, 2007. Elevated risks of pregnancy complications and adverse outcomes with increasing maternal age. Hum Reprod 22:1264-72.

Macassa G, Ghilaber G, Bernhardt E, Burstrom B, 2004. Contribution of household environment factors to urban childhood mortality in Mozambique. East Afr Med J 81:408-14.

Mané NB, Simondon KB, Diallo A, Marra AM, Simondon F, 2006. Early breastfeeding cessation in rural Senegal: causes, modes, and consequences. Am J Public Health 96:139-44.

Mesike CG, Mojekwu JN, 2012. Environmental determinants of child mortality in Nigeria. J Sustainable Dev 5:65-75.

Miotti PG, Taha TET, Kumwenda NI, Broadhead R, Mtimavalye LAR, Van der Hoeven L, Chiphangwi JD, Liomba G, Biggar RJ, 1999. HIV transmission through breastfeeding. A study in Malawi. J Am Med Assoc 282:744-9.

Mosley WH, Chen LC, 1984. An analytical framework for the study of child survival in developing countries. Popul Dev Rev 10:25-45.

Musafili A, Essén B, Baribwira C, Binagwaho A, Persson L-Å, Selling KE, 2015. Trends and social differentials in child mortality in Rwanda 1990-2010: results from three demographic and health surveys. J Epidemiol Comm Health 0:1-7.

Nduati R, John G, Mbori-Ngacha D, Richardson B, Overbaugh J,
Mwatha A, Ndinya-Achola J, 2000. Effect of breastfeeding and formula feeding on transmission of HIV-1: a randomized clinical trial. J Am Med Assoc 283:1167-74.

Niragire F, Wangombe A, Achia TNO, 2011. Use of the shared frailty model to identify the determinants of child mortality in Rwanda. Rwanda J 20:89-105.

NISR, 2006. Geodata: district boundaries. National Institute of Statistics of Rwanda, Kigali, Rwanda.

NISR, 2015. Rwanda poverty profile report: integrated household living conditions survey 2013/14. National Institute of Statistics of Rwanda, Kigali, Rwanda.

NISR, MINECOFIN, 2014. Rwanda fourth population and housing census 2012. Thematic report on population size, structure and distribution. National Institute of Statistics of Rwanda, Kigali, Rwanda.

NISR, MOH, ICF International, 2011. Rwanda demographic and health survey 2010. NISR, Ministry of Health and ICF International, Calverton, MD, USA.

Norton M, 2005. New evidence on birth spacing: promising findings for improving newborn, infant, child, and maternal health. Int J Gyn Obs 89:51-6.

Nyandekwe M, Nzayirambaho M, Kakoma JB, 2014. Universal health coverage in Rwanda: dream or reality. Pan Afr Med J 17:232.

Omariba DWR, Beaujot R, Rajulton F, 2007. Determinants of infant and child mortality in Kenya: an analysis controlling for frailty effects. Popul Res Pol Rev 26:299-321.

Ombok M, Adazu K, Odhiambo F, Bayoh N, Kiriinya R, Slutsker L, Hamel MJ, 2010. Geospatial distribution and determinants of child mortality in rural western Kenya 2002-2005. Trop Med Int Health 15:423-33.

Orji E, Shittu A, Makinde O, Sule S, 2004. Effect of prolonged birth spacing on maternal and perinatal outcome. East Afr Med J 81:388-91.

Rabbi AMF, 2012. Mass media exposure and its impact on fertility: current scenario of Bangladesh. J Sci Res 4:383-95.

Reidpath DD, Allotey P, 2003. Infant mortality rate as an indicator of population health. J Epidemiol Commun Health 57:344-6.

Rutstein SO, Johnson K, 2004. The DHS wealth index. DHS Comparative Reports No. 6. ORC Macro, Calverton, MD, USA.

Rutstein SO, Rojas G, 2006. Guide to DHS statistics. Demographic and health surveys methodology. ORC Macro, Calverton, MD, USA.

Saksena P, Antunes AF, Xu K, Musango L, Carrin G, 2011. Mutual health insurance in Rwanda: evidence on access to care and financial risk protection. Health Policy 99:203-9.

Sastry N, 1997. Family-level clustering of childhood mortality risk in Northeast Brazil. Popul Stud 51:245-61.

Schafer JL, 1999. Multiple imputation: a primer. Stat Methods Med Res 8:3-15.

Schoeps A, Gabrysch S, Niamba L, Sie A, Becher H, 2011. The effect of distance to health care facilities on childhood mortality in rural Burkina Faso. Am J Epidemiol 173:492-8.

Sekabaraga C, Soucat A, Diop F, Martin G, 2011. Innovative financing for health in Rwanda: a report of successful reforms. Yes, Africa can: success stories from a dynamic continent. World Bank Publications, Washington, DC, USA.

Selemani M, Mwanyangala MA, Mrema S, Shamte A, Kajungu D, Mkopi A, Mahande MJ, Nathan R, 2014. The effect of mother's age and other related factors on neonatal survival associat- 


\section{Article}

ed with first and second birth in rural, Tanzania: evidence from Ifakara health and demographic surveillance system in rural Tanzania. BMC Pregnancy Childbirth 14:240.

Singh A, Pallikadavath S, Ram F, Alagarajan M, 2013. Do antenatal care interventions improve neonatal survival in India? Health Policy Plan 29:842-8.

Spiegelhalter DJ, Best NG, Carlin BP, Linde AVD, 2002. Bayesian measures of model complexity and fit. J Roy Stat Soc B 64:583-639.

Sutcliffe AG, Barnes J, Belsky J, Gardiner J, Melhuish E, 2012. The health and development of children born to older mothers in the United Kingdom: observational study using longitudinal cohort data. Brit Med J 345:e5116.

UN, 2005. The millennium development goals report 2005. United
Nations, New York, NY, USA.

UN, 2012. The millennium development goals report 2012. United Nations, New York, NY, USA.

UN, 2014. The millennium development goals report 2014. United Nations, New York, NY, USA.

UN, 2015. Transforming our world: the 2030 agenda for sustainable development. United Nations, New York, NY, USA.

Victora CG, Huttly SR, Fuchs SC, Olinto MTA, 1997. The role of conceptual frameworks in epidemiological analysis: a hierarchical approach. Int J Epidemiol 26:224-7.

WHO, 2013. Consolidated guidelines on the use of antiretroviral drugs for treating and preventing HIV infection. Recommendations for a public health approach. WHO Library Cataloguing-in-Publication Data, Geneva, Switzerland. 